Research Article

\title{
Developing an Extended IFC Data Schema and Mesh Generation Framework for Finite Element Modeling
}

\author{
Zhao Xu $\mathbb{D}^{1}{ }^{1}$ Zezhi Rao, ${ }^{1}$ Vincent J. L. Gan, ${ }^{2}$ Youliang Ding, ${ }^{1}$ Chunfeng Wan (D), \\ and Xinran Liu $\mathbb{D}^{1}$ \\ ${ }^{1}$ Department of Civil Engineering, Southeast University, Nanjing 210096, China \\ ${ }^{2}$ Department of Civil and Environmental Engineering, The Hong Kong University of Science and Technology, Hong Kong, China
}

Correspondence should be addressed to Zhao Xu; bernardos@163.com

Received 5 May 2019; Revised 21 July 2019; Accepted 6 September 2019; Published 3 October 2019

Academic Editor: Giovanni Garcea

Copyright (c) 2019 Zhao Xu et al. This is an open access article distributed under the Creative Commons Attribution License, which permits unrestricted use, distribution, and reproduction in any medium, provided the original work is properly cited.

\begin{abstract}
Mesh generation plays an important role in determining the result quality of finite element modeling and structural analysis. Building information modeling provides the geometry and semantic information of a building, which can be utilized to support an efficient mesh generation. In this paper, a method based on BRep entity transformation is proposed to realize the finite element analysis using the geometric model in the IFC standard. The h-p version of the finite element analysis method can effectively deal with the refined expression of the model of bending complex components. By meshing the connection model, it is suggested to adopt the method of scanning to generate hexahedron, which improves the geometric adaptability of the mesh model and the quality and efficiency of mesh generation. Based on the extension and expression of IFC information, the effective finite element structure information is extracted and extended into the IFC standard mode. The information is analyzed, and finally the visualization of finite element analysis in the building model can be realized.
\end{abstract}

\section{Introduction}

There is a global trend in evaluating the overall performance of buildings by means of the building information model (BIM) among structural design participants, such as designers, fabricators, and construction managers. However, the building models store the complete geometry of a construction, and they are not directly adequate for numerical simulation, as they only implicitly describe the topology and the mutual connections of different structural components [1]. In order to make the semantic data as well as the connection explicit and elaborate interoperability among building design participants, initiatives are put forward by extending the Industry Foundation Classes (IFC) model, which represent a promising approach toward ensuring software interoperability in the building industry. IFC standard extensions to the data model provide three extension mechanisms: extensions based on adding entity definitions, extensions based on IfcProxy entities, and extensions based on attribute sets. Users can refer to the model architecture of the IFC standard system and extend the IFC data model according to their actual needs. Treeck and Rank [2] developed an algorithm based on the graph theory for analyzing and interpreting building model-based geometry in order to support CAD (computer-aided design) tools with numerical simulation techniques, which are applicable to any building energy simulation tool. Furthermore, this relation obtained by the developed spatial and topological analysis may serve as the basis for queries by means of a spatial query language for BIMs. Nevertheless, another problem arose that despite various simulation applications are available for analysis, data input from CAD drawings cannot be efficiently and precisely read by those applications. To solve this problem, Fazio et al. [3] designed an IFC-based framework for implementing the performance evaluation protocol. To refine the IFC instance files, Ying and Lee [4] proposed a rule-based automatic checking approach dedicated to the validation of IFC 2nd level-proper space boundaries for energy analysis. Similarly, a technologytransfer model named shared computer-aided structural 
design (SCASD) model is developed, affording a new methodology of interaction, namely, electronic interaction (e-interaction) [5]. All of these studies have expanded the use of IFC standards in different areas.

What is equally worth discussing is that, to fully explore the three-dimensional structure model described by the IFC, the finite element method (FEM) is introduced to the structural analysis. A triangle mesh is a common object representation adopted in scientific computing [6] as well as animation [7] adopted in scientific computing, but the accuracy of triangular mesh calculation in finite element calculation is not as high as that of the quadrangle mesh. While mesh generation is extremely crucial for the accurate FEM analysis and computation, Lee and Lee [8] detailed about how to design and develop a full-quadrilateral finite element mesh generator (Graph FEM V1.0). However, Nagakura et al. [9] mentioned that hexahedral meshes have obvious advantages in terms of calculation error, the number of cells, and resistance to distortion, so hexahedral meshes are becoming a mainstream mesh in FEM analysis. Lai et al. [10] improved this proposal to identify different many-toone sweepable volumes to generate hexahedral elements. In addition, to be more specific, Miranda and Martha [11] described a hexahedral mesh generation algorithm ideally suited for transition subdomain meshes in the context of any domain decomposition meshing strategy. The algorithm decomposes hierarchically a volume until all volumes should be mappable to generate elements by the $3 \mathrm{D}$ mapping approach. Based on the generation of hexahedral mesh, Elhaddad et al. [12] presented a numerical discretization technique for solving 3-dimensional material interface problems involving complex geometry with the multilevel hp-FEM. By using a separate hexahedral mesh for each material subdomain and applying weak constraints on the interface conditions between different meshes, the weak discontinuity of the material interface is solved.

Considering the three-dimensional modeling techniques and the meshing methods in mind, it should focus on how to overcome the gap between BIM and FEM in building structural analysis. In this paper, attempts have been made to develop an effective extended IFC schema and mesh generation framework to improve the architectural model meshing and finite element modeling. Section 2 briefly discusses the trends of research and the current range of applications of BIM and FEM. Section 3 introduces the theoretical methods involved in this paper and shows the complete process of the whole project implementation. In Section 4, the case study is discussed and the research methods and future research direction are analyzed.

\section{Literature Review}

2.1. IFC Standard. The application of BIM in the construction industry to enhance productivity has become widespread. Open BIM data schemas include the Industry Foundation Classes (IFC) [13] and the green-building XML schema. Among these two most popular BIM schemes, gbXML and IFC, IFC appears to be a suitable choice as it is richer in content, enables interoperability among different software environments, and can be updated according to the building's modifications [14]. With the rising adoption of BIM, a range of research studies have focused on IFC, the official data schema of BIM, to fully utilize and analyze the BIM data. Each IFC object contains its individual boundary representation model (BRep model), from which the attributed objects of the building can be derived via the IFCEurostep Toolbox in combination with the geometric modeler ACIS from Spatial Corp [1]. However, Krijnen and Beetz [15] stated that the objectified relationship model and low-level nature of IFC render many interesting relationships implicit, such as spatial connectivity and type information. Therefore, additional inference steps are often applied to increase its ease of use, querying efficiency, interoperability, and the discoverability of IFC information.

A wide variety of promising attempts have been proposed to develop the application and to improve the interoperability of IFC. To extend the interoperability of a construction quality database in the evaluation process by employing IFC, Xu et al. [16] proposed integrated solutions to improve current quality management processes with the assistance of an IFC-based working environment. By expanding the property set of IFC data, nontraditional building information is embedded into the BIM to build a mapping relationship between BIM information and external database information. Another aspect is noticed that at present, getting the data out of BIM is still a hard task, and no BIM authoring tool able to properly export 2nd level space boundary geometry information contained in IFC can be found. To conquer this problem, Lilis et al. [17] proposed an approach to extract such information correctly, which is necessary for the setting up of building energy simulations.

In previous studies, BIM data standard still has many shortcomings in dealing with multiprofessional collaboration. Each major only involves information related to the major and does not implement the process of extracting complete data. As a result, the uniform IFC format does not work as well as it should, and it is difficult to achieve synergies between different application levels. Such problems are not unrecognized. To handle this problem, this research focused on defining a schema using popular relational database structure following an established star schema model and on transformation rules to bring BIM data into a form that is easily queried. Likewise, Daum and Borrmann [18] described a system for topological and spatial querying on IFC building models. Elements intersecting or contained within boundaries of other elements can be retrieved. Kang and Hong [19] developed an automatic mapping method to improve the efficiency of IFC data conversion process to CityGML, aiming at the problem that the mapping model in BIM and GIS conversion process takes more time to calculate.

2.2. Mesh Model. At present, the technology of grid segmentation is improving, and the precision and applicability of grid segmentation are increasing and expanding. The successful applications of the use of the information model as a standard practice for buildings led to an increase in 
demand for its use in other structures as well. In addition, there is neither IFC supports road structures nor specific road BIM execution plan exists. Consequently, a continuous active effort has been made to develop an IFC-based data schema for effectively representing the design information of civil engineering structures other than buildings for productivity improvement. Yabuki [20] proposed the IFCshield Tunnel and included items for representing components, facilities, and geologic information targeted at shield method tunnels. Thereafter, Lee and Kim [21] proposed an IFC data schema expansion plan targeting integrated road structures, which include bridges and tunnels. Nevertheless, these studies focused more on the relationship from a macroperspective and from a holistic perspective, respectively, rather than considering the elements of the tunnel structure in detail. Lee et al. [22] presented a tunnel data schema to efficiently manage and exchange the design information, by identifying the applicable IFC elements and defining new items for the additional necessary elements. Similarly, Park et al. [23] put forward a method to improve the usability of the IFC data schema-based bridge information model that can control not only the 3D geometric information but also nongeometric attributes for an open standard-based bridge information model at present.

Besides above application and enhancement of both IFC and BIM authoring tools, another problem arose when dealing with the cooperation of BIM and FEM. At present, there is only a few of studies on cooperation of BIM and FEM [24]. Xiao et al. [25] analyzed the combined parametric method of BrIM models, built up structural properties for FEM, and highlighted that the most essential factors for finite element analysis are element types, material properties, mesh modes, and restraint system. Moreover, as for FEM, Romberg et al. [1] discussed the advantage of the fully three-dimensional structural analysis performed by p-version of the finite element analysis. The main method adopted in this paper is to establish the BRep entity geometric model for mesh generation, which is due to the unique advantages of the BRep geometric model in the relationship between points, lines, and planes representing the structure. Huang et al. [26] proposed the geometric topology model of CSG-BRep to describe the three-dimensional topological relationship of the structure, aiming at the limited topological information expressed by the BRep geometric model. This paper introduces the data structure of the BRep model in detail and unifies the topological relationship between models by using the Boolean operator.

The literature reviewed above concludes a wide variety of promising approaches proposed to enhance the interoperability and the discoverability of IFC information. Nevertheless, attempts have to be made to further overcome the limitations imposed by lack of consistency in data and the declining interoperability in the field of building construction [22]. IFC is a data model that relies on human interpretation in the sense that the meanings of its modeling elements are not constrained by means of formal semantics, leading to calls for such a formalization of IFC in some formal language to be frequently used for computational reasons [27]. Thus, developing a method to transfer the architecture model to the finite element model effectively is the key to fully utilizing and analyzing BIM data. The data transformation can provide effective support for the finite element analysis process of IFCformat BIMs created on the web browser. By using the web technology, BIMs can be linked with external information like FEM-data from other systems on the internet to improve the visualization performance.

\section{Methodology}

The traditional transformation from the architectural model to the structural model is usually to establish a general structural finite element model and to convert the general structural analysis model to the architectural model, which has a very good effect in the past when the interaction between architectural and structural model is not strong. With the development of BIM technology, the interaction degree of the building structure model is continuously improved. The direct finite element analysis of the building model can better realize the use of a model in multiple aspects, which greatly facilitates the management of building information. Moreover, there are a series of incompatibility problems in the transformation between the traditional general structural analysis model and the architectural model, and the mismatch of the model after the transformation, which are also the shortcomings exposed by the traditional transformation method.

In this study, a novel mesh segmentation method is proposed to support the data conversion from the BIM to the finite element model. The proposed method first transforms a given IFC-based BIM into a connection model, which is then meshed to obtain a refined finite element analysis model for the subsequent calculations and simulation. Then, the meshed model is used to set boundary conditions and load, and the finite element analysis is carried out. In the process of grid division, the first thing is to ensure the extraction of components based on the whole model so as to facilitate the view of the analysis results of components. Then, the relative coordinate and local coordinate transformation of the components are defined, and the components are meshed using the refined grid algorithm, at last the coordinate data of the unit section are encoded and compiled. The information transformation about nongeometric attributes, especially the loads and constraints, generally exists in the model. If these nongeometric attributes cannot be compiled in the model, the nongeometric information under IFC standards should be embedded in the original geometry file. At the same time, this paper also expanded and expressed the IFC attribute set and processed CAE results with WebGL technology combined with compressed data to facilitate the calculation means and results of finite element analysis to be applied and displayed in the BIM. By processing the results, the control of line frame display and selection of display result list can be achieved. In addition, the selection and display position control of color bar can also be achieved. After calculating the data of the finite element model, it is able to display the results of finite element analysis in the BIM. Figure 1 shows the proposed BIM-based framework for mesh generation. 


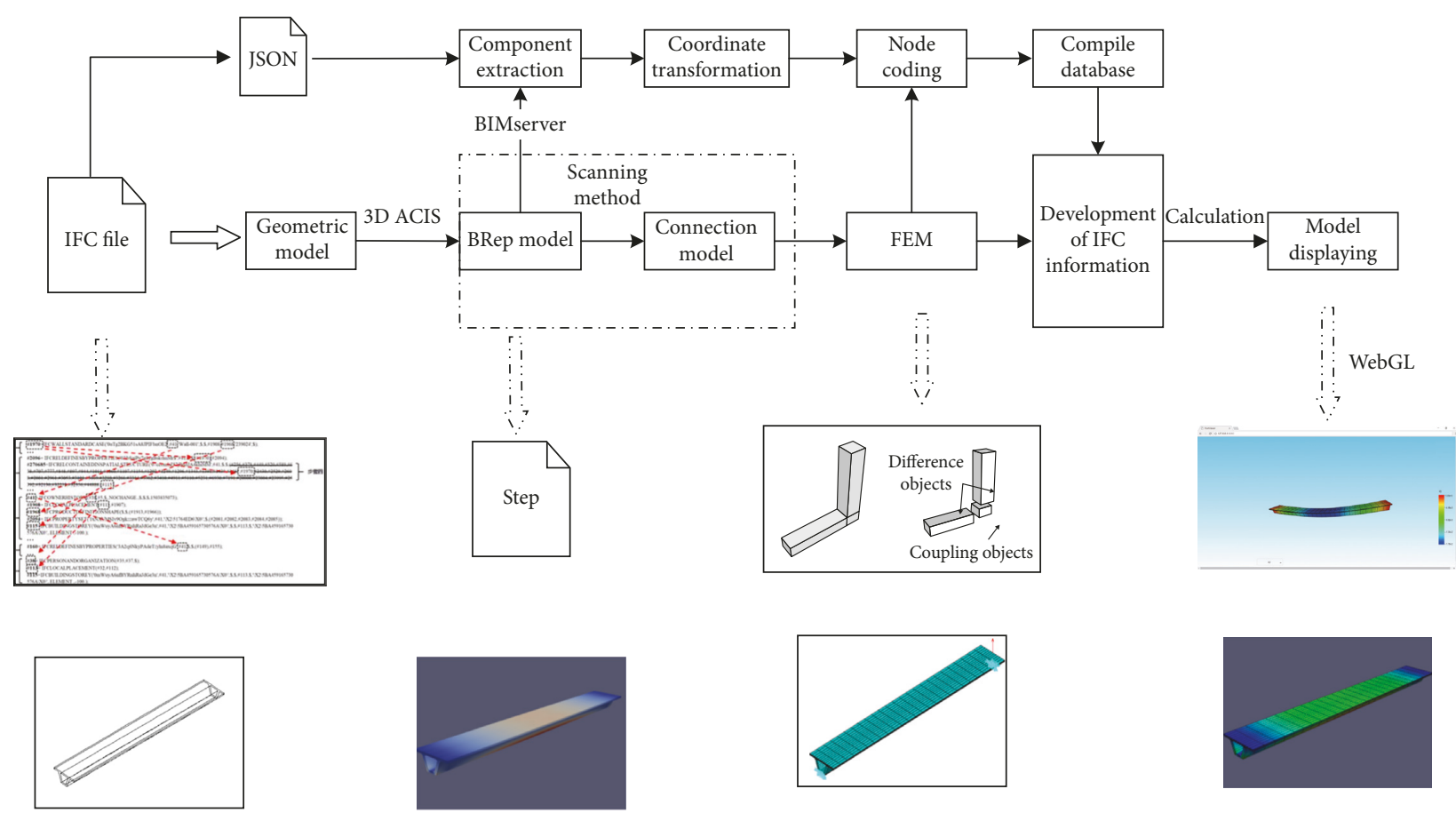

Figure 1: Proposed BIM-based framework for mesh generation.

\subsection{Transformation Method}

3.1.1. hp-Vision Finite Element Method. The transition between architectural and structural models is influenced by many factors. For traditional architectural models, the collection method based on scanning volume and construction entity is generally adopted [26]. In the surface model, BRep (boundary representation) model can be used to express commonly [28]. In theory, the classical finite element method (h-vision) is very suitable for the simulation of 1,2 , and 3 dimensional structural problems [29]. However, it has certain requirements on the aspect ratio of the structure shape, and low-precision locking may occur when the case of plate and shell structure is encountered [1]. For simple materials, the grid generator can follow the material page exactly, but it can be cumbersome at the composite interface. In order to avoid too complex mesh, a p-version finite element method which extends the original complex geometry to simple shape through the virtual domain is introduced into the finite element analysis method so that the cartesian mesh can be divided easily. While using the $\mathrm{p}$-vision, it can well simulate the complex boundary. However, materials with complex geometric shapes need to obtain local high gradients or singular points, and the problem must refine the local grid, which greatly increases the space occupied by the hard disk. A multilevel hp-finite cell method is proposed to enhance the structural analysis model to be more accurate in finite element expression. The hp-vision finite element method combines $\mathrm{h}$-vision and p-vision, which can deal with complex $3 \mathrm{~d}$ geometry. This scheme is especially suitable for dealing with discontinuity of interface of different materials.

With the improvement of users' aesthetic requirements, attention has been paid to the appearance and style of architecture, and more complex curved surfaces and irregular shapes have been applied to architecture. The finite element method based on hp-vision combination is adopted to establish smaller grid surface at the microlevel of the structure and achieve more accurate effect through continuous subdivision so as to express the architectural information.

3.1.2. Model Transformation Algorithm Based on BRep Bodies. In a simple basic structure described by the IFC files, it mainly contains the following contents:

(1) The basic content of the project, which is mainly provided by IfcProject:

IfcOwnerHistory. Creator, application, and creation time

IfcGeometricRepresentationContext. Type, dimension, coordinate system, precision, etc.

IfcUnitAssignment. Unit of measure

(2) The basic spatial structure of the project, which is mainly provided by IfcBuilding:

IfcLocalPlacement. Describe the relative spatial position of an artifact

IfcProductDefinitionShape. Define geometry in the local coordinate system

In the geometric representation of the model, the IFC standard provides several methods: construct solid model, construct solid geometry model (CSG model), surface model, subdivision surface model, and boundary representation model. The CSG model has the advantages of simple method, fast generation speed, simple data structure, small data volume, and easy modification. CSG is the result of a Boolean operation in which an object is represented as a 
series of simple elementary objects (such as cubes, cylinders, and cones). The disadvantage of CSG is that the information is simple and does not record the boundary, vertex information, and so on. Limited by the type of basic voxel and the type of voxel operation, it indicates that the coverage area of the body has great limitations and the local operation of the body (e.g., chamfering) is not easy to realize. The space required to display the resulting shape represented by CSG is also longer. The scanning representation, also known as the stretch body representation, represents the two-dimensional contour drawn on the working plane and then stretches the contour in a certain direction to form a three-dimensional object. The advantage of scan representation is that it is simple and suitable for graphic input means. The disadvantage of the scanning representation is that it is difficult to perform geometric transformation, the boundary information of the shape cannot be directly obtained, and the coverage of the shape representation is very limited. The surface model is a series of two-dimensional faces enclosed into a three-dimensional form, which is expressed in a more direct way without too much node information. The subdivision surface model is composed of a triangular mesh, which is more detailed in expression than the surface model.

An object of the BRep model is represented as a region of closed space connected by many surfaces such as faces, triangles, and splines. The advantage of boundary representation is that there is more information about faces, edges, points, and their relationships. It is good for generating and drawing wireframe and projection diagram and easy to be associated with 2-dimensional drawing software and surface modeling software. The disadvantage of boundary representation is that because its core information is surface, it cannot provide information about entity generation process. The BRep model needs more information to describe the object, and the expression form of the boundary expression method is not unique. The BRep model has great advantages in finite element mesh segmentation because the solid model contains many relationships between points and lines.

\subsection{From BIM to Finite Element Model}

3.2.1. Model Transformation Algorithm Based on BRep Objects. In recent years, the development of engineering collaborative design has become more and more urgent. Collaborative design requires a high degree of integration of data and information to avoid inefficient and repetitive work. In other words, when the building scheme is determined, the data information of the building model created can be extracted by the downstream participating majors (structure, energy saving, equipment, etc.) to form corresponding data model, so as to reflect the concept of collaborative design. At present, IFC is generally used in the data exchange of building models in the construction and facility management industry. The goal of this product model standard is to define an integral, object-oriented, and semantic model of all components, attributes, properties, and relationships of and within a building model. The IFC product model is specified using the modeling language EXPRESS, which has been used to define STEP-based product models within ISO 10303 before. Since 2014, the IFC4 standard has been released.

The methods of describing solid in IFC standard are introduced, which mainly include scanning solid model, surface model, boundary representation model, solid geometry model, and subdivision surface model. Although the IFC standard building model can well express the geometric shape of a complete structure, it is not suitable for numerical simulation because it only implicitly expresses the topological structure and mutual relations between different components. It is difficult to describe the connection of structures using the general stretching method in IFC.

In order to make the topology and connection of the structure explicit, it should further express the geometry of the building. In this case, it needs to establish the BRep model of the object with attribute objects. BRep is a method of using boundary surface slices to express entities. The entity object expressed by BRep is surrounded by many small faces. Each facet is composed of many boundary lines. Each boundary line is formed by connecting two vertices. The orientation of each face can be judged by its normal direction. The advantage of using BRep technology is that there is much information about points, edges, planes, and their mutual relations, and it is beneficial to generate and draw wireframe, projection diagram, and so on. However, its main disadvantage is that the boundary form is arbitrary and not unique, which is not conducive to the development of the data conversion interface.

In order to be able to correctly express the connection and topology of the model, a BRep-model with attributed objects is derived from the building model firstly. As the second step, the geometric model is decomposed into "connection model" which includes coupling objects and difference objects. The coupling objects are set in all location in which structural components are arranged in plane contact, while difference objects only being in contact with other difference objects at nodes or along common edges. In the third step, the model is divided into finite element meshes by scanning solid quadrilateral meshes, as shown in Figure 2. The advantage of this model is that it not only completes the mesh division of the model but also decomposes the beam-slab-column wall effectively.

3.2.2. Connection Model Definition. Consider a consistent geometric building model as a set of one or more BRep bodies.

The following types of intersection of objects can be distinguished in Figure 3:

(i) Type PLF. The intersection of objects with adjacent faces consists of nodes, edges, and faces

(ii) Type PL. The intersection of objects with adjacent edges consists of nodes and edges

(iii) Type $P$. The intersection of objects with adjacent nodes consists of nodes only 


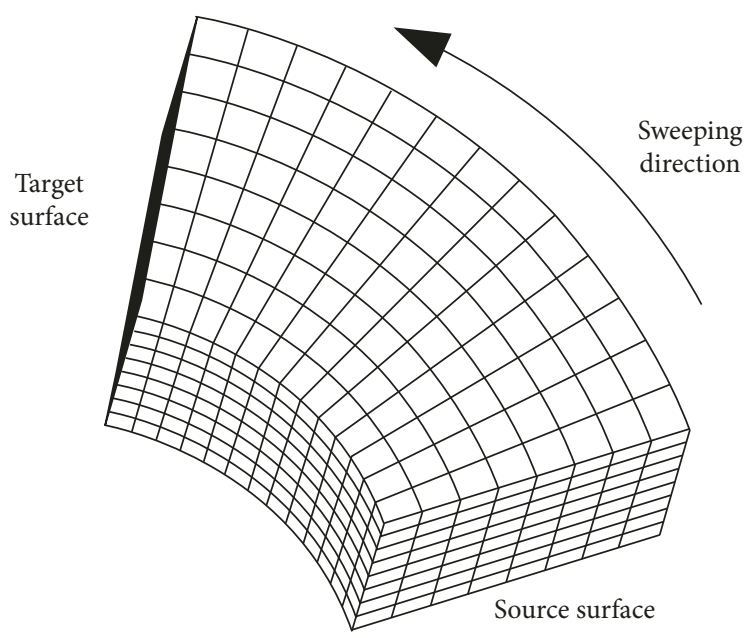

Figure 2: Principle of the sweeping mesh division.

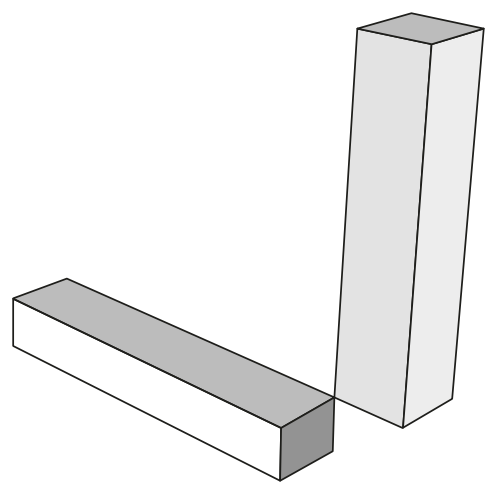

(a)

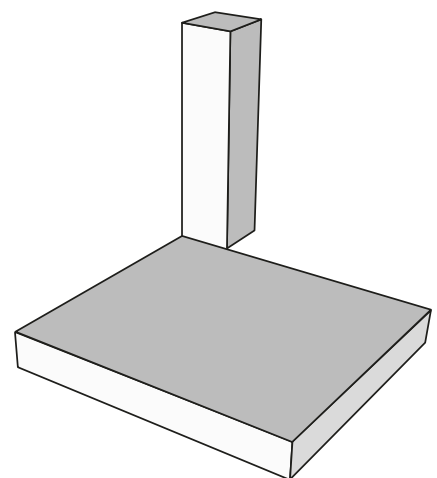

(b)

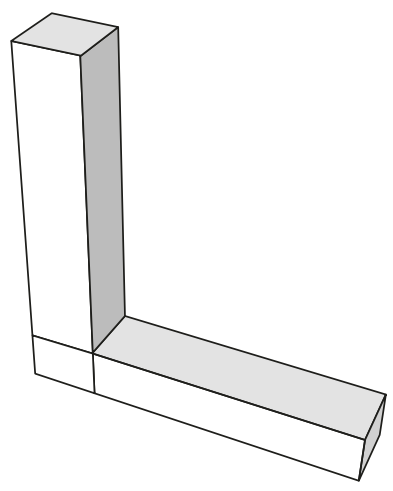

(c)

Figure 3: Intersection types: (a) P, (b) PL, and (c) PLF.

Objects can thus be characterized according to their intersections with other objects. Based on their different semantics, they are partitioned into a set of coupling objects MK and into the set of difference objects MD (Figure 4).

Each coupling object and difference object is composed of closed BRep objects being described by nodes, edges, and faces. The connection forms between difference objects are types of P or PL. The connections between coupling objects are types of P, PL, or PLF. The connection forms between the difference object and the coupling object are types of P, PL, or PLF.

To consider a geometric building model $\Omega \subset R^{3}$ consisting of a set of $n$ BRep objects, the algorithm to decompose the entity model into join models is shown in Algorithm 1, and the process is shown in Figure 5.

\subsubsection{Automatic Mesh Generation with Hexahedral Elements.} After the BRep model expressed the connection relationship of the model correctly, it needs to start meshing the structure. The initial point of mesh generation is the model that is decomposed using the algorithm in the previous section. Coupling and difference objects are meshed with explicit given sequences and type-sensitive meshing algorithms. It can either use elementary $3 \mathrm{D}$ mesh macros or hexahedral meshes scanned using a 2D quad mesh. Firstly, it has to define a reasonable mesh size for each different object. The most critical element in the partitioning process is to generate compatible elements. Only when the position of nodes, edges, and faces of hexahedra on coupling objects is inherited to their adjacent difference objects, elements without hanging nodes can be guaranteed.

When the model is divided into coupling object and difference object, the hexahedral meshing is generated. In view of the characteristics of building structure shape rules and easy to establish efficient model, this paper adopts the generation method of the hexahedral grid based on twodimensional quadrilateral grid scanning. In this paper, a method of generating hexahedral meshes based on twodimensional quadrilateral meshes is proposed, aiming at the characteristics of building model shape rules and easy to build efficient models. In this method, an improved leading edge advance algorithm is used for two-dimensional quadrilateral meshing, and sweep method is used for threedimensional meshing. The meshing process is discussed in [2]. Multilevel hp-finite cell method was used to generate 

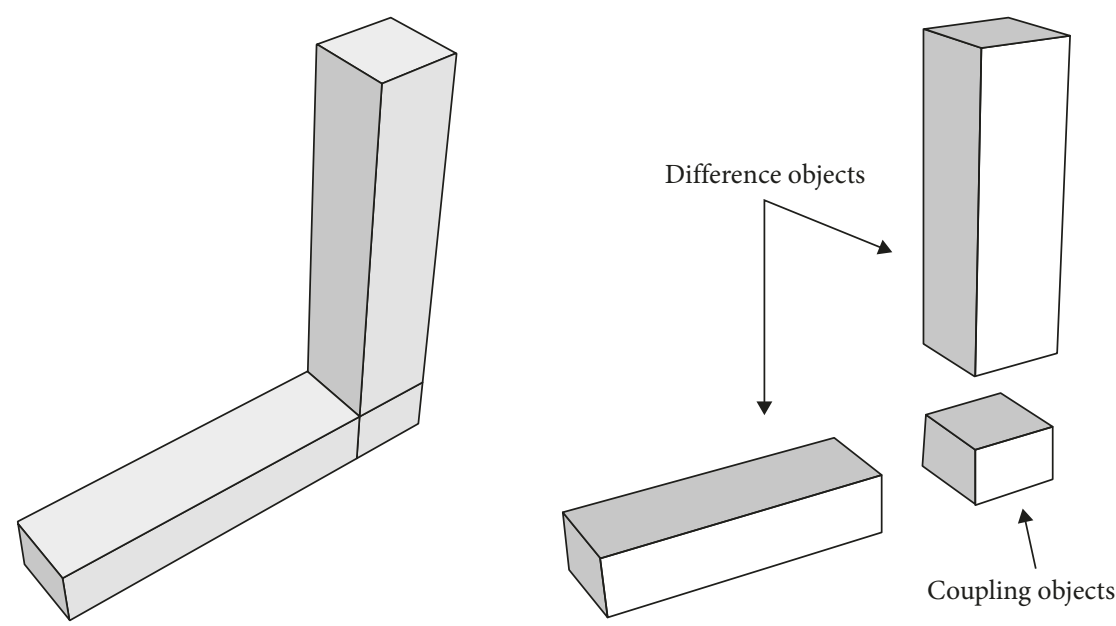

FIGURE 4: Connection model with coupling and difference objects.
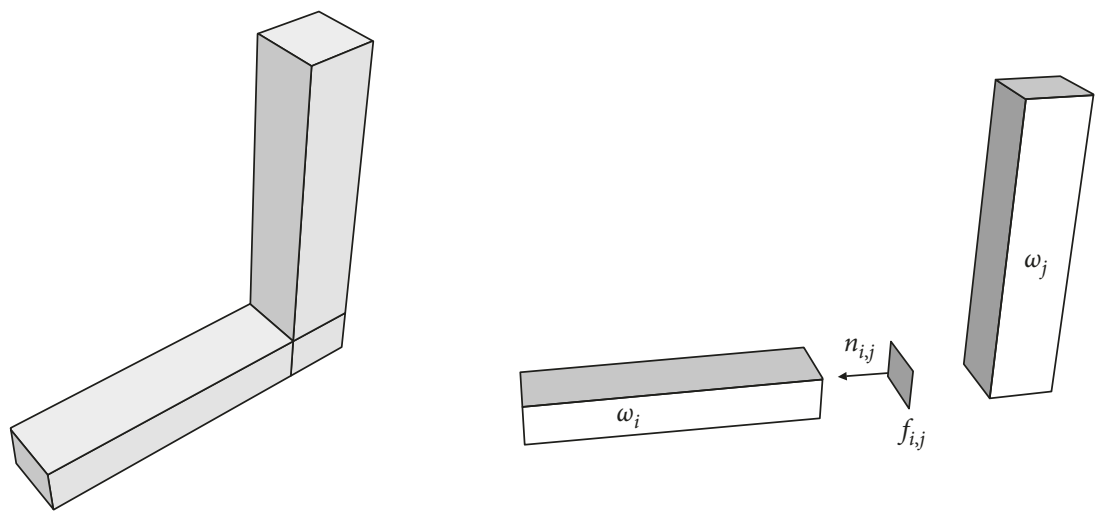

FIGURE 5: Connection model with coupling and difference objects.

meshes of different sizes for the division of source surface meshes.

In the sweep method, the requirements for the model are as follows: first, the source surface and the target surface must have the same geometric topology. Second, the source and target surfaces may not be two-dimensional, but they must be parameterized. Third, the connecting surface must be a quadrangle-like closed surface. Last, the meshing of the connecting surfaces must be structured.

After sweeping a single component, it is transmitted through the coupling objects and difference objects between the connectome. In the process of grid division, the size and shape of each component are different, so it is generally necessary to transfer the grid through the same boundary. The transfer process is as follows: (1) Determine the element density in the sweeping direction, which is determined according to the fineness of the finite element mesh; (2) Determine the sweeping advance vector of the quadrilateral grid by combining the hexahedral transition template. Because there are different sizes of meshes in the process of mesh generation, different advance vectors should be set up for mesh segmentation; (3) Use the sweep method to generate internal nodes layer by layer and generate hexahedral elements. For the quadrilateral grid element nodes of the two-dimensional source surface, the corresponding sweep advance vector is adopted to generate the internal nodes layer by layer, and then the hexahedral elements are generated layer by layer according to the geometric topological relations of the hexahedral elements and their transition templates; (4) Restore of missing hexahedral mesh transition elements. Hexahedral transition elements cannot be completely divided by the sweep method, and a certain number of mesh elements will be missing in the transition grid divided by the sweep method. After scanning, some nodes are repaired.

The process of meshing between connectors is as follows: (1) Use the grid generator to cut the difference objects by the sweeping method, and then sweep in the third direction; (2) Assign values through common face coupling objects, and then refine the coupling object; (3) Pass through the common surface to the adjacent difference object. In this process, only when the position of nodes, edges, and faces of hexahedra on coupling objects is inherited to their adjacent difference objects, elements without hanging nodes can be guaranteed. If the size of the hexahedra mesh is different, the coupling part needs to be refined again, and part of the node information is no longer transferred. For mesh refinement, the formation 
process of the connectome grid is shown in Figure 6. H-p finite element mesh division can well maintain the uniformity and continuity of the geometric gradient and improve the efficiency of mesh discretization.

3.2.4. Finite Element Information Extraction Based on the IFC Standard. A building is composed of many components. When analyzing a single component, it should extract the component information and then divide the structure into grids. The method of grid partitioning is given above. There are many basic structures for grid partitioning, and they are often used together on a case-by-case basis. Hexahedral element has significant advantages in finite element analysis, so the hexahedral element should be used as far as possible in the construction of the finite element model. The size of the element is determined according to the accuracy of the calculation. It also needs to collect the element body information in order to complete the finite element analysis of the building model.

For a unit hexahedron, the coordinate information of the point is the main concern. After extracting the component information, the frame point coordinates of the component are found. The point coordinates of the hexahedron can be defined through geometric relation. In order to obtain the coordinates of each point, the first step is to determine the coordinates of the frame point of the component. The extraction of coordinate information of frame points requires nested references of multiple IfcLocalPlacement objects so as to obtain the geometric positioning of the local coordinate system of components in the absolute coordinate system. After obtaining the absolute coordinates of the frame points, the relative coordinates and absolute coordinates of each point can be obtained through the geometric relationship between the nodes in the process of mesh segmentation. The shape expression of the IFC model is different from that of component structure. It is necessary to calculate the topology information of each node of the element body according to the shape expression of the structural component and the location of the local coordinate system. In the finite element analysis of the whole structure, for the frame beam and column, the two ends of their topological expression are the two end points of the beam and column in the analysis model. For the wallboard component, the corner points expressed by its topology are the corner nodes in the analysis model.

(1) Component Extraction Based on the IFC. In the building model, many building components are continuous beams, cross-story columns, and cross-story walls, which need to be split up and establish the relationship between structural components and structural connections when generating structural components. Before the finite element analysis, it should extract the components in the IFC file first and then divide the components into grids. The internal data structure based on IFC standards uses algorithms to extract independent components. According to the types of building components selected by users, such as walls, beams, and columns, the target components are extracted from IFC physical files. In order to express the extracted IFC files of components correctly, the algorithm is required to extract not only the physical information of components but also other nonphysical information.

The IFC physical files are preprocessed first. The algorithm is used to detect the IFC instances corresponding to the target building artifacts, and then the circular iteration is used to extract the examples of the associated link with the target instance. During this period, redundant instances are eliminated until all relevant instances are extracted. Finally, the extracted IFC files are processed after processing, and the IFC files are checked for validity. The whole procedure flow is shown as Figure 7.

The BRep components extracted in the process are stored in IFC files as physical files. The IFC nongeometric information is written or accessed in the conversion process. If the nongeometric information is not shown in the standard physical files or is missing, the physical files need to be supplemented accordingly. The BRep geometry model, as the intermediate state of the running program, is contained in the IFC files.

(2) Local Coordinate System Analysis. Structural components (including other entities in the structural analysis model) refer to the unified coordinate system of the structural analysis model, and the hierarchical coordinate reference method in the architectural model is not adopted. The structural components are expressed by topological geometry so as to distinguish the connection relations efficiently. Therefore, the transformation between local coordinate systems should be considered in the expression process.

The unified coordinate system of the structural analysis model does not use the hierarchical coordinate reference method in the building model, so the coordinates need to be converted. The IFC standard stores the position coordinate information of the object through the IfcLocalPlacement type. In IFC files, spatial structure is defined by IfcRelAggregates. The location of the model component is defined by local position. Each component has individual independent coordinate system (IfcLocalPlacement). Attribute PlacementRelTo corresponds to the coordinate system of an upper order, and attribute RelativePlacement corresponds to the information of relative position. Then, through multiple references of the coordinate system, the coordinate system of components is nested under multiple IfcLocalPlacement, and its position in global coordinate system of the whole project is finalized. Through nested references of multiple IfcLocalPlacement objects, the relative position is obtained of the local coordinate system of components in the global coordinate system (the localization of the origin and the axes of local coordinate system in global coordinates). The position of the object does not point the world coordinate system but points a relative object in the local coordinate system. For example, the position of a wall is determined by the local coordinate system of an adjacent beam which is set in the overall coordinate system of the entire building. All objects have precise position coordinates in the world coordinate system, so the coordinate system must be converted during the mapping. Hence, the conversion of geometric information 


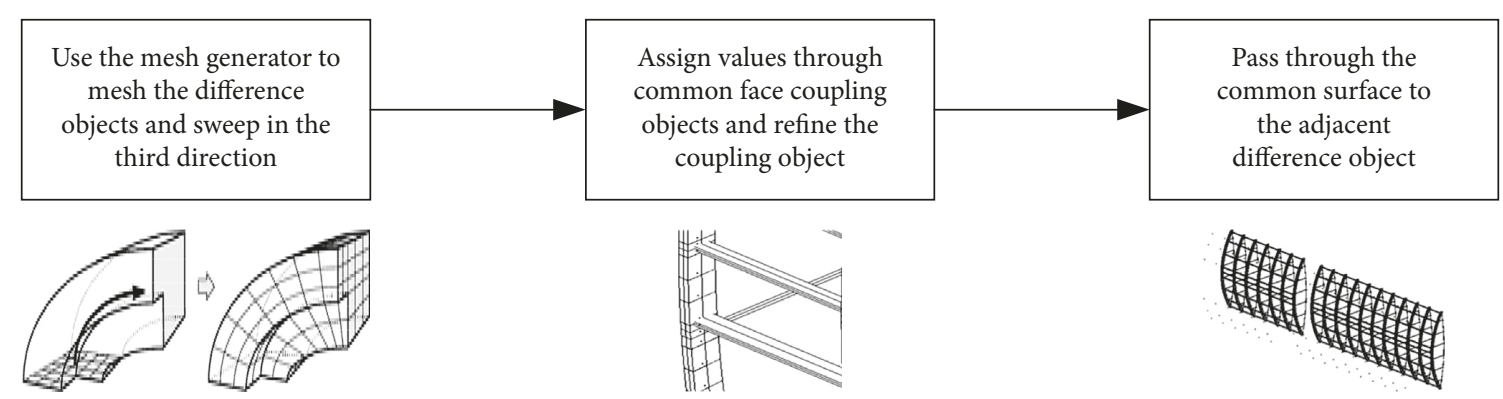

FIgURE 6: The meshing process.

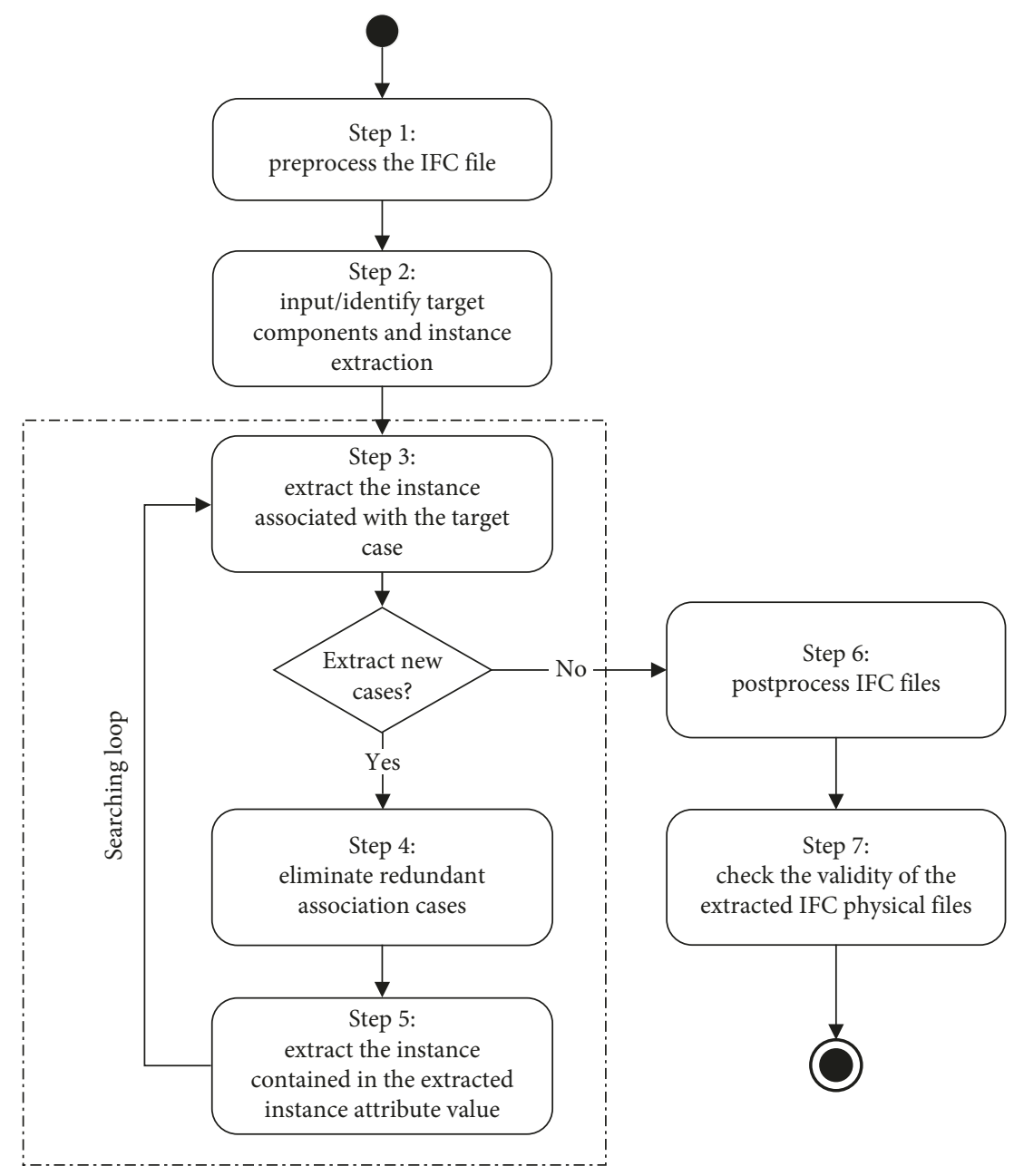

FIgURE 7: Component extraction based on IFC physical files.

from IFC contains the transformation of the coordinate system. Upon model display, the coordinate system employed of model is the local cartesian coordinate system. BIMserver is a BIM-based server management platform. This platform can be used as an example. Upload the IFC file to the BIMserver server, parse the model through IFCEngine, split the entire model according to the building components, and obtain its component model (IFC file). After the above process, the original IFC file is converted into a smaller IFC file obtained by the component and a
JSON file containing the component properties. In the process of extracting components, it is necessary to traverse the various components in the IFC file, obtain the geometric positioning, shape expression, and related material properties of the local coordinate system of the component in the entire model coordinate system, and store them in a JSON file. Generally, the origin of coordinates is on the surface of ellipsoid and corresponding elevation zero. The calculation of transition matrix $T_{t}$ between two coordinate systems is as follows: 


$$
\begin{aligned}
\mathbf{T}_{\mathbf{t}} & =\mathbf{R}_{x} \mathbf{R}_{y} \mathbf{R}_{z} \mathbf{T}, \\
\mathbf{R}_{x} & =\left[\begin{array}{cccc}
1 & 0 & 0 & 0 \\
0 & \cos \theta_{x} & \sin \theta_{x} & 0 \\
0 & -\sin \theta_{x} & \cos \theta_{x} & 0 \\
0 & 0 & 0 & 1
\end{array}\right], \\
\mathbf{R}_{y} & =\left[\begin{array}{cccc}
\cos \theta_{y} & 0 & -\sin \theta_{y} & 0 \\
0 & 1 & 0 & 0 \\
\sin \theta_{y} & 0 & \cos \theta_{y} & 0 \\
0 & 0 & 0 & 1
\end{array}\right], \\
\mathbf{R}_{z} & =\left[\begin{array}{cccc}
1 & 0 & 0 & 0 \\
0 & \cos \theta_{z} & \sin \theta_{z} & 0 \\
0 & -\sin \theta_{z} & \cos \theta_{z} & 0 \\
0 & 0 & 0 & 1
\end{array}\right], \\
\mathbf{T} & =\left[\begin{array}{cccc}
1 & 0 & 0 & 0 \\
0 & 1 & 0 & 0 \\
0 & 0 & 1 & 0 \\
x_{1} & y_{1} & z_{1} & 1
\end{array}\right],
\end{aligned}
$$

where it is assumed that the origin of the local coordinate system in the IFC model is $\left(x_{1}, y_{1}, z_{1}\right)$ in the global coordinate system; the $Z$-axis of local coordinate system is perpendicular to the ground; the $Y$-axis points to due north; the spin matrix corresponding to coordinate axes is $\mathbf{R}_{x}, \mathbf{R}_{y}$, and $\mathbf{R}_{z}$; and $\theta_{x}, \theta_{y}, \theta_{z}$ are the intersection angles between rotation vector and $X$-axis, $Y$-axis, and $Z$-axis.

In the process of meshing the local components, the coordinate information of the physical instance should be mapped to the world coordinate system, and only by mapping to the unified world coordinate system can the measurement and comparison be made according to the same standard. The geometric representation of the IfcProduct instance is based on the local coordinate system, and the local coordinate system in the world coordinate system will be affected by the reference coordinate system. To obtain the representation of its local coordinate system in the world coordinate system, follow the reference coordinate system of IfcProduct and trace upward until its PlacementRelTo is empty. This paper takes the coordinate transformation between a beam and a column as an example to illustrate the method of calculating the world coordinate system. The column section is $500 \times 500 \mathrm{~mm}$, the height is $3600 \mathrm{~mm}$, the beam section is $500 \times 300 \mathrm{~mm}$, and the length is $2000 \mathrm{~mm}$. The instance fragment is shown in Figure 8 .

(3) Calculation of Node Coordinates Based on IFC Standard. A node is the most basic finite element entity. A node describes a physical location on the created structure and is used by the element to define the location and shape. It can also be used as a temporary input to create geometric objects. The three-dimensional finite element model element can be understood as a three-dimensional body composed of several nodes, and each three-dimensional element is

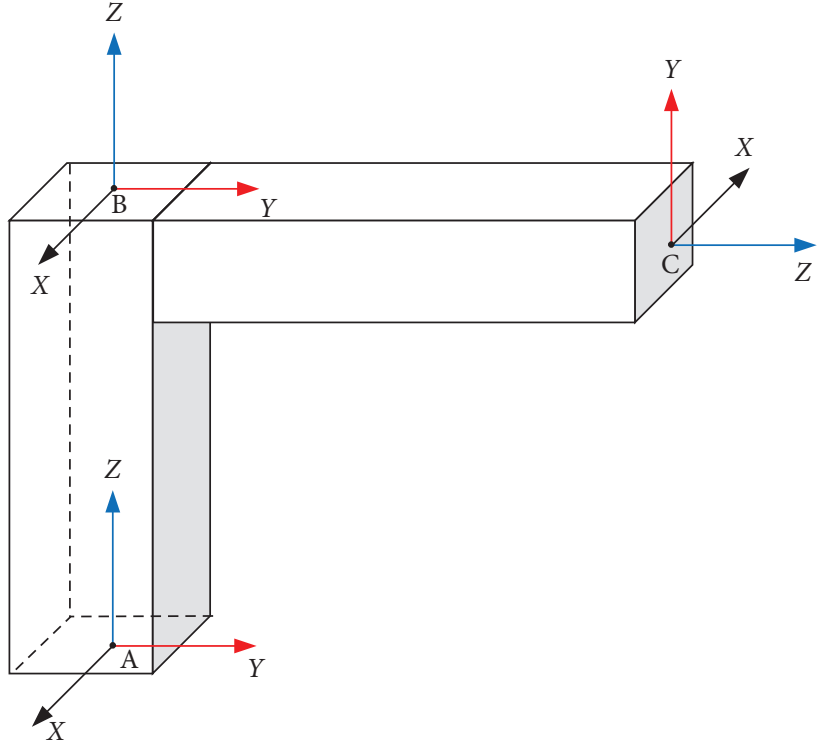

Figure 8: Coordinate system transformation in IFC physical files.

connected to form a complete finite element model. Therefore, if the nodes in each finite element model are created and then connected together according to a principle, the finite element model can be created.

In the process of assembling components in the point graph, it needs to extract the component information firstly and then obtain the position information of the frame points of the component in the absolute coordinates from the IFC standard information so as to calculate the position information of each point in the IFC context. According to the solution method above, the absolute coordinate system of each component, the reference coordinate system of each component, and the relative position of the absolute coordinate system are obtained after nesting layer upon layer. In the process of finite element mesh segmentation, the mesh elements involved include one-dimensional node element, two-dimensional quadrilateral element, and three-dimensional hexahedron element. The data structure of node cell and tetrahedral cell is simple. No matter what kind of grid can be represented by nodes arranged in a certain order. The quadrilateral element can be represented by its four nodes in order. For the purpose of unifying the data of the subsequent hexahedral element, it is stipulated that the node order of the quadrilateral element should conform to the right-hand rule. The data structure of nodes is similar to that of geometric points. The data structure of the quadrangle includes two parts, element number (Id) and element node set (Elemet2D_Nodes). The data structure of nodes and quadrilateral cells can be specifically expressed in Algorithm 2.

The hexahedral element is composed of six quadrilateral elements in geometric topological relation, so a hexahedral element can be represented by 8 nodes of two relative quadrilateral elements. These eight nodes must be one-to-one in space. The data structure of the hexahedral element includes the hexahedral number (Id) and the eight nodes of the hexahedral element (Element3D_Nodes). Its data structure is expressed in Algorithm 3. 
After the nodes of the hexahedral element are defined, the coordinates of the base points of the components can be found by looking up the coordinate information in the IFC file, and then the coordinate values of the other points can be calculated. Firstly, a rectangular coordinate system for the component is established, and the origin is the base point of the component. According to the method of meshing mentioned above, it can be found that the coordinates of all components are in the coordinate system of the component. Tetrahedrons, hexahedrons, triangular prisms, and pyramid elements can be used in conjunction with complex, irregular entities. With the calculation above, the graph of all node positions and coding information can be formed. Then with consideration of the load information, the position change information is obtained as the standard for structural analysis of components.

3.2.5. The Extension of Nongeometric Properties. The structural analysis model mainly includes nodes, units, loads, and constraints. The IFC standard provides a comprehensive set of predefined entities for structural analysis, in which the relevant finite element elements are prefixed with IfcStructural. For the node connection, IfcStructuralConnection is mainly used in the IFC standard to express the connection nodes between elements in the structural model. The connection here mainly refers to the connection node or support concept of the structural analysis model (finite element model); the structural analysis model of the unit with IfcStructuralMember entity to express, IfcStructuralMember have two child class, don't is the line unit (IfcStructuralCurveMember) and surface single yuan (IfcStructuralSurfaceMember); for the structure's load representation in IFC, there are multiple load conditions and multiple load combinations according to the design specifications. Therefore, accurate expression of the load groups to the key. The combination of IfcStructuralAction and IfcStructuralLoadGroup is generally adopted to express the load condition and load combination; structural analysis model of constraint expression using IfcBoundaryCondition entities. This entity can express support boundary constraints (applies to IfcStructuralConnection) and can express the end of the bar to degrees of freedom (for use in IfcRelConnectsStructuralMember). About beam section includes point about beam (IfcBoundaryNodeCondition), edge (IfcBoundaryEdgeCondition), and face about about bouquets (IfcBoundaryFaceCondition). The writing or access of this nongeometric information in IFC standard needs to be reflected only in the process of transformation. If it is not reflected in the standard physical file or there is lack of information, the physical file needs to be supplemented accordingly.

3.2.6. Establishment of the IFC-Based Finite Element Model. In the process above, the BIM is subjected to the requirements of the finite element analysis model. To realize the finite element analysis of BIM, on the one hand, it needs to expand and express the finite element information of the processed BIM; on the other hand, the mapping relationship between data and model is integrated, as shown in Figure 9. In this way, the information of the finite element model is firstly extended in IFC standard, and then the finite element information is integrated into the BIM.

(1) The Extensions Based on IfcProxy Entities. As an open standard system, the IFC standard provides three extension mechanisms for the extension of the model, namely, extension based on IfcProxy entity, extension by adding entity definition, and extension based on attribute set. Each of the three extension methods has its own advantages and disadvantages, and different methods can be used for different users. For example, if the national standard is formulated, entity expansion is required, and the relevant attribute sets are supplemented. For project-level BIM applications, the IfcProxy extension or attribute set extension can be used. Compared with the extension method based on the attribute set, the operation is simple and convenient, the version compatibility is good, the type is safe, and the operation efficiency is high, which is the preferred expansion method.

In the IFC standard, an entity can have multiple attribute sets. These attribute sets can be either a predefined attribute set or a custom attribute set, which lays the foundation for us to extend the attribute set. The association of an entity with its attribute set is achieved by defining an attribute relationship entity. As a container of attribute information, the attribute set loads the structural force information that the engineers care about.

This paper first establishes a basic information attribute set about a finite element body and defines the attribute content contained in the attribute set. When defining the content of an attribute, it needs to determine both the attribute type and the attribute value type. Usually the attribute type is IfcpropertySingleValue. After the definition of the basic information attribute set and related attributes of the structure is completed, the identification information attribute set at the structural component level is established, including the relative and absolute coordinate information of the individual points of the unit body. Since beams, columns, slabs, and walls of structural components all have these two attributes, in the actual extension, the common parent class of IfcBeam, IfcColumn, IfcSlab, and IfcWall, IfcBuildingElement can be selected as the associated entity of the extended attribute set, which is also a reflection of the flexibility of IFC standards in practice. The rating and rating value properties of beam, column, board, and wall are included in the Pset_AppraisalResult property set, and the rating and rating values in the property set are defined.

After the definition of component attribute set and related attributes is completed, the specific method of definition in the process of grid generation should be defined to ensure the accuracy of information expression as much as possible in the compilation process. The grid attributes of components are defined as shown in Table 1.

The set of encoding attributes of a cell body is shown in Table 2. 


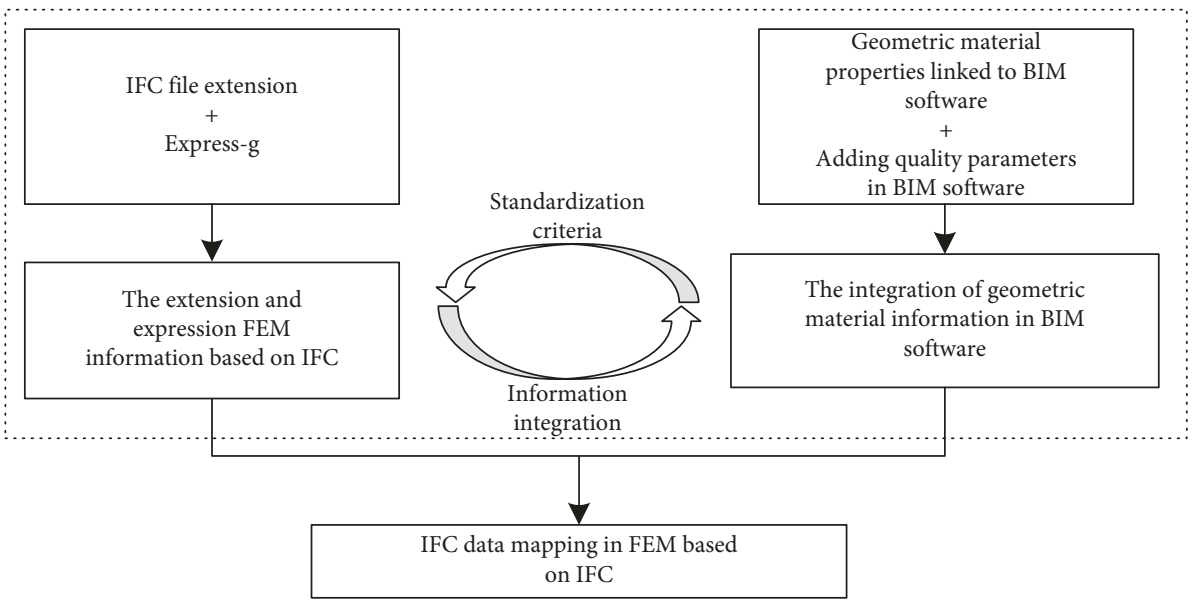

FIGURE 9: Implementation of IFC data mapping in the FEM model.

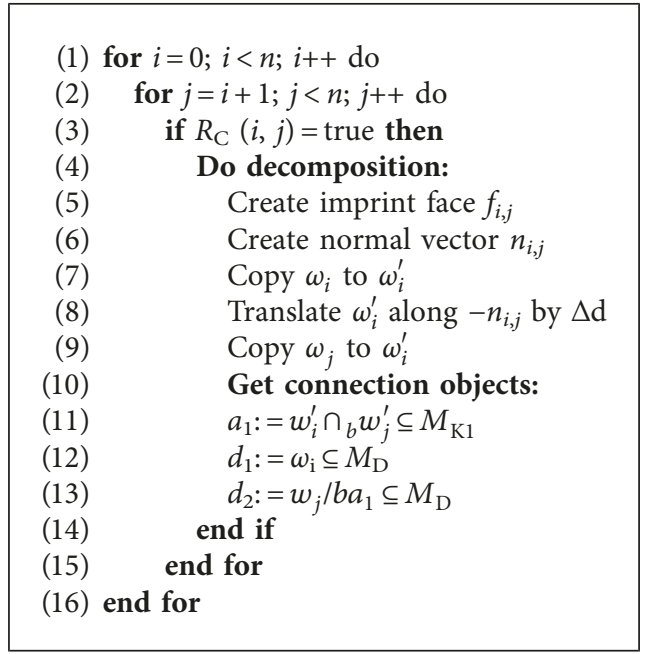

Algorithm 1: Decomposition into the connection model.

\author{
Type Mesh_Node \\ Id as Long \\ Position3D (0 to 2) as Double \\ End Type \\ Type Mesh_Element2D \\ Id as Long \\ Element2D_Nodes (0 to 3 ) as Mesh_Node \\ End Type
}

Algorithm 2: Data structures for nodes and quadrilateral cells.

After the expansion of the attribute set, it is required to express the finite element analysis model based on IFC standard further. IFC standard data need to be expressed in EXPRESS normalized language, which provides two ways of description: EXPRESS language description in code form and express-g diagram in tree form (a graphical representation of the product model corresponding to the EXPRESS language, known in the standard as a "graphical subset of the EXPRESS language"). Because the steps of manually modifying the code form of EXPRESS language are tedious, the workload is large, and it is easy to make mistakes. Therefore, after the IFC standard is extended a little according to the user's own needs, it is often described by the express-g diagram to complete the expression of the extension information. The EXPRESS language description in the code form is mostly used after the IFC standard system is updated or other IFC standards are extensively extended and modified. In this paper, IFC-based finite element analysis 


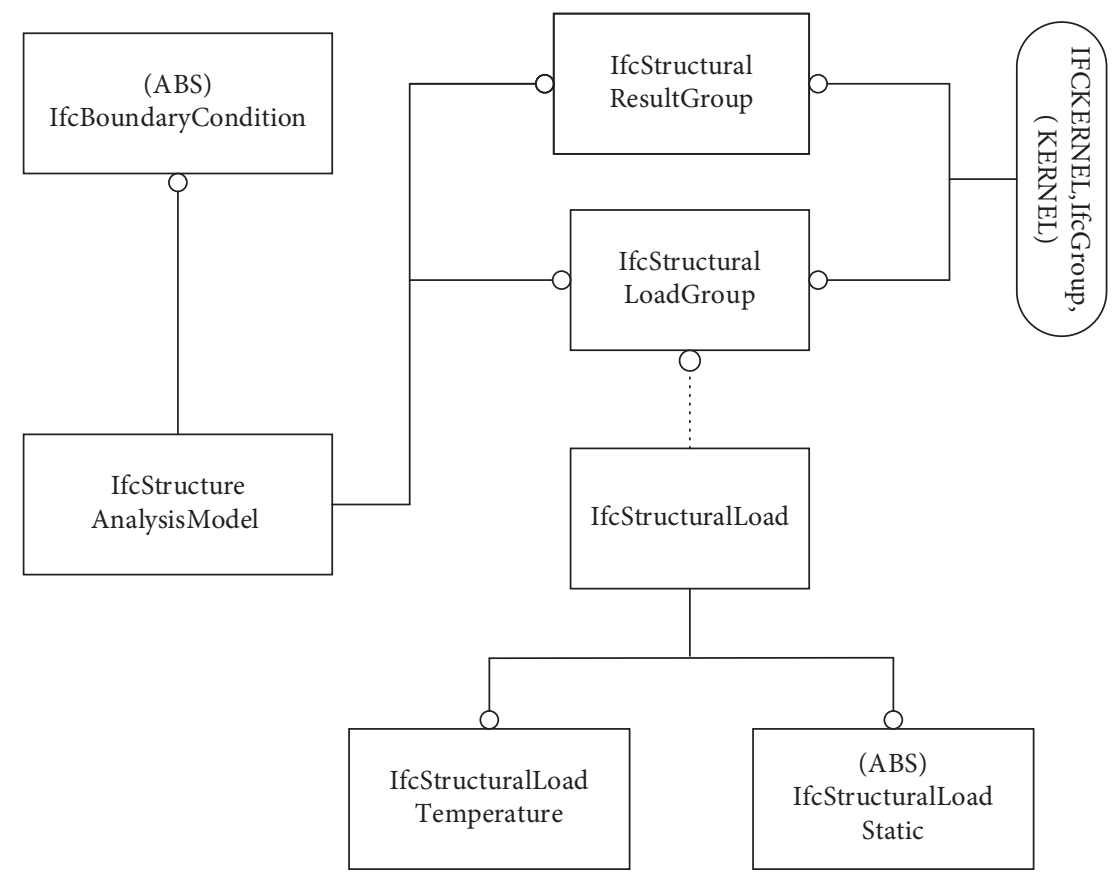

FIgURE 10: The express-g diagram description of IFC entities.

information extension did not define new entities, but extended based on attribute set. The express-g diagram is selected here to describe the quality information extended above as shown in Figure 10.

In Figure 11, among the five types of entities, IfcProduct, IfcElement, IfcBuildingElement, IfcPropertySetDefinition, and IfcPropertySet, the two adjacent types of entities are inheritance relations, which are expressed with bold solid lines and circles pointing to subclass entities. An association relationship is established between IfcPropertySet and IfcEntity through IfcPropertySetDefinition so that the construction quality status of IfcEntity can be expressed through the construction quality evaluation information contained in IfcProperty. IfcProperty includes two subclass entities, IfcComplexProperty and IfcSimpleProperty, of which IfcSimpleProperty includes six subclass entities. The set of properties defined in this article is the optional set of properties of the entity IfcBeam/Column/Slab/Wall (which inherits all the properties of IfcBuildingElement), connected by a dotted line. Figure in the contents of the elliptic dotted box is defined as 4 item properties: OnsiteConcreteBeam/Column/Slab/explicit attribute of the Wall, through the thin lines is connected to a set of properties. The attribute values of the four attributes can be divided into enumerated values and simple values according to their types, and the relationship between them and the attribute value entities is mandatory association, which is connected by thin solid lines. The symbol "(ABS)" represents abstract class, which is an entity that cannot be directly displayed in the construction project, and exists so that other entities can appear as its superclass.

(2) Database Preparation. In order to make the data storage, identification, association, call, and other management work in the database more convenient and orderly, it is necessary to encode the relevant data, which should follow the principles of standardization, simplicity, uniqueness, and integrity. In addition to the above coding principles, during the finite element analysis and calculation, the node and element number will have an impact on the bandwidth and the number of wave front segments of the total stiffness matrix of the structure and then on the calculation time and database capacity. At the present stage, there are many optimizers that can improve the node number twice, and the node number and unit number are more conducive to calculation. In order to facilitate coding, the coding order of nodes is carried out according to the righthand rule, and the grid in the $X Y$ plane is corresponding to the adjacent one in the $Z$ direction, and so on, to reach the coding information of each point. After finite element analysis of the structure, a large amount of data will be generated, which is not conducive to data processing. In this study, it is proposed to lightweight the scalar data, vector data, and grid data in the results, compress the data by using compression algorithm, and improve the efficiency of displaying the results.

(3) Information Transfer between Database and BIM Software. For the data to be used in the finite element analysis of the BIM, in addition to the coordinate information and element information of some sections and nodes contained in the IFC file mentioned above, the material information and load information of the structure are also included. In Figure 12, it describes how this information is connected to the entire model through the express-g language. After encoding the node information of components, the finite element information of each component into the database is compiled. The processes are as follows: 


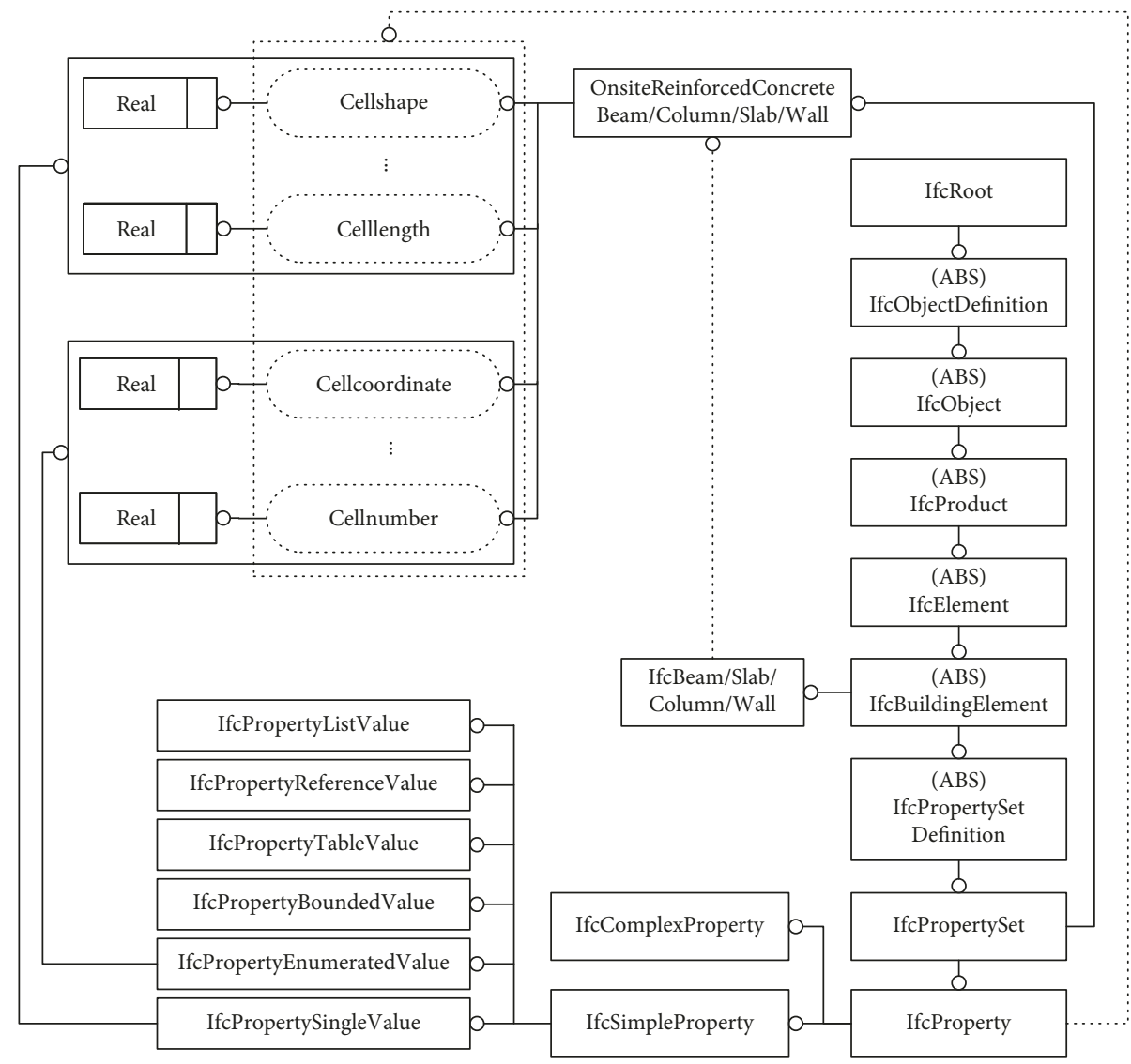

FIgURE 11: Express-g diagram for finite element analysis.

(i) Replace the component instance with the corresponding absolute coordinate information

(ii) Define the component attribute coding

(iii) Enter the information about all artifacts

(iv) Associate the absolute coordinates of a component with its corresponding attribute information

(v) Postprocess and check the database information, validity check

(vi) Associate database with IFC file

When conducting finite element analysis on the structure, first of all, it should locate the coordinate information of the origin of the relative coordinate system of the structural components in the absolute coordinate system. After finding the coordinate system of the basic points of the components, the database should be traversed to find all the information related to the finite element analysis of the relevant nodes. The processes of extracting coordinate information are as follows:

(i) Preprocess IFC file

(ii) Input, recognition, and absolute coordinate extraction of target components

(iii) Traverse the database to identify absolute coordinate information corresponding to the target artifact (iv) Extract information about mechanics, materials, lengths, and other correlations associated with absolute coordinates

(v) Extract database information postprocessing, check validity

Through the above process of database preparation, the finite element analysis point information can be obtained. The obtained information is exported to the simulator for calculation, and the obtained results are fed back to the model for display.

\section{Discussion}

4.1. Case Study. In Figure 13, a simply supported beam bridge, which is box girder type, is taken as a sample. First, the BIM is based on IFC standard to generate the BRep entity model. Then, the model is meshed, and the results of meshing are exported to the dataset. The database is established according to the way of component arrangement. Through the finite element analysis, the cloud image of the structure under stress can be obtained. Such results can be displayed in the BIM software, and the stress and strain conditions of each point can be obtained so as to achieve the visualization of the finite element analysis of the BIM. The deadweight, vertical live load, and surface load are considered in the structural analysis in Table 3 . The stress 

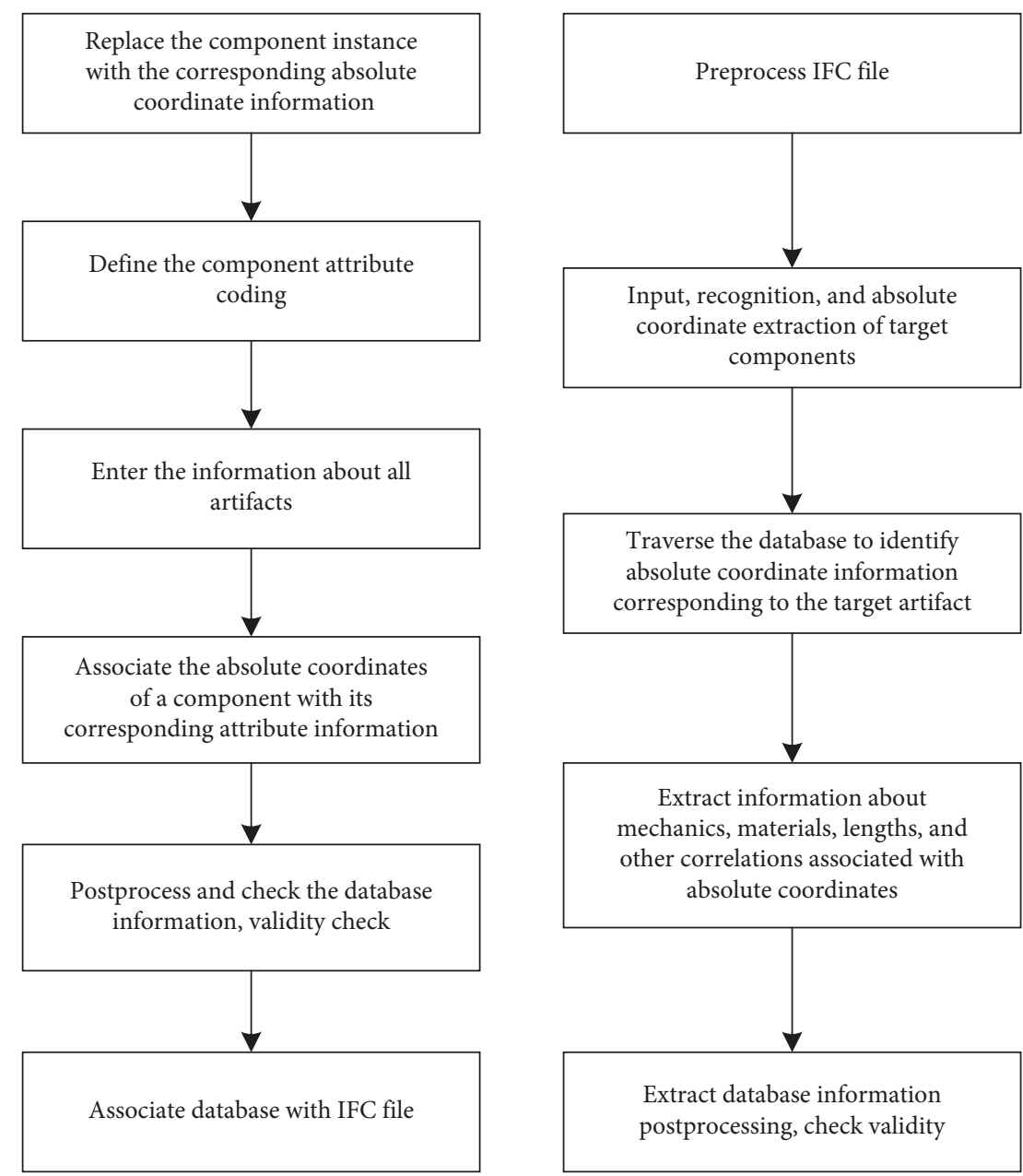

FIGURE 12: Database compilation process.
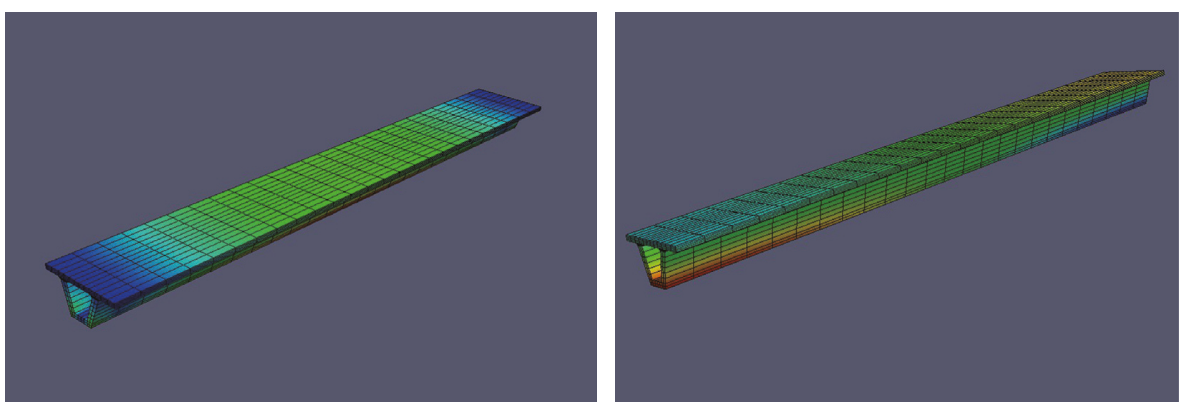

FIGURE 13: Generation of mesh and display of finite element analysis results.

Type Mesh_Element3D
Id as Long
Element3D_Nodes ( 0 to 7 ) as Mesh_Node
End Type

Algorithm 3: Data structure of a hexahedron.

and strain analysis of the beam under deadweight is calculated, and the node information is compared, and the effect diagram is generated.
WebGL technology can be used to perform CAE presentation of the finite element calculation results of the bridge. In addition, users can choose to display a list of 
TABLE 1: Grid attributes of components definition.

\begin{tabular}{llcc}
\hline Attribute name & Attribute type & Attribute set type & Attribute definitions \\
\hline CellShape & IfcpropertySingleValue & Boolean & Shape of element \\
CellLength & IfcpropertySingleValue & Boolean & Length of element \\
\hline
\end{tabular}

TABle 2: Cell body information definition.

\begin{tabular}{|c|c|c|c|}
\hline Attribute name & Attribute type & Attribute set type & Attribute definitions \\
\hline CellCoordinate & IfcpropertySingleValue & Boolean & Coordinate information \\
\hline CellNumber & IfcpropertySingleValue & Boolean & Coded information \\
\hline $\mathbf{U}_{x}$ & IfcpropertySingleValue & Boolean & Displacement of $\mathrm{X}$-axis \\
\hline $\mathbf{U}_{y}^{\prime}$ & IfcpropertySingleValue & Boolean & Displacement of Y-axis \\
\hline $\mathbf{U}_{z}$ & IfcpropertySingleValue & Boolean & Displacement of $\mathrm{Z}$-axis \\
\hline Usum & IfcpropertySingleValue & Boolean & Integrate displacement \\
\hline
\end{tabular}

TABle 3: Parameter information of the bridge.

\begin{tabular}{lllll}
\hline $\mathrm{E}\left(\mathrm{N} / \mathrm{m}^{2}\right)$ & Section shape & Length $(\mathrm{m})$ & Surface load $(\mathrm{kPa})$ & Deadweight per cubicmetre $\left(\mathrm{kg} / \mathrm{m}^{3}\right)$ \\
\hline $3.25 \times \mathrm{e}^{10}$ & 25 & 500 & 2600
\end{tabular}

results that can be viewed both on the surface and inside of the component. The results of the cloud image displayed can be switched by selecting the color bar and control the position. The strain cloud image in the specific direction can be selected based on the effects to facilitate the more detailed analysis of the structure. Figure 14 shows the node displacement cloud image of $\mathrm{U}_{y}$ and sum directions based on the structure on the website.

Figure 15 shows the deformation of beam-column connections under the uniformly distributed load. Under the different mesh densities, the beam and column mesh segmentation are also transmitted through the coupling joint. The analysis results of the structure can be displayed and manipulated on the web side as the cases above.

4.2. Technology Comparison. At present, in the finite element analysis of BIM, there are two main technologies in continuous implementation and application. One way to achieve the goal is to use finite element analysis software to enhance the application of building models. Now some commercial software claims that they have developed related software applications and platform systems. However, this kind of platform is more based on the expansion of the product chain of its own software and does not have strong universality. In contrast, the IFC standardbased BIM introduced in this paper is more versatile in the development of finite element analysis. In the finite element analysis based on the IFC standard, some studies develop the IFC standard interface directly. Through data transformation, the building model can be better generated and imported into the structural model for calculation, which is also a relatively simple and convenient way at the present stage. In the studies so far, direct analysis of IFC files for structural analysis has not received more attention. The IFC mesh segmentation method in this paper can effectively calculate the information of each point in the finite element mesh segmentation. Other information such as material information, load information, and node information is stored in a database according to the EXPRESS language standard for computing purposes. Because the grid segmentation of components is based on IFC standards, when the finite element calculation of these nodes is carried out, the results can be directly displayed in the BIM.

4.3. Future Work. Although the finite element analysis of the structure by using the BRep model is effective, there are still a lot of work to be improved in the future, including the following:

(1) Improvement of Algorithms about Meshing Need. The boundary constraint condition for constructing harmonic scalar fields in our algorithm, namely, the source surface and the target surface mentioned in this paper, is constructed by the user through interactive selection tool on the boundary surface of the swept body. This not only increases the user's inconvenience but also leads to the large deformation difference between the source surface and the target surface, which is not conducive to the generation of high-quality hexahedral mesh. In the future, it is proposed to consider using the surface segmentation method and shape analysis technology on the surface grid of the model to automatically divide the surface of the model into surface regions with the same characteristics, and then automatically set boundary constraint conditions of harmonic 

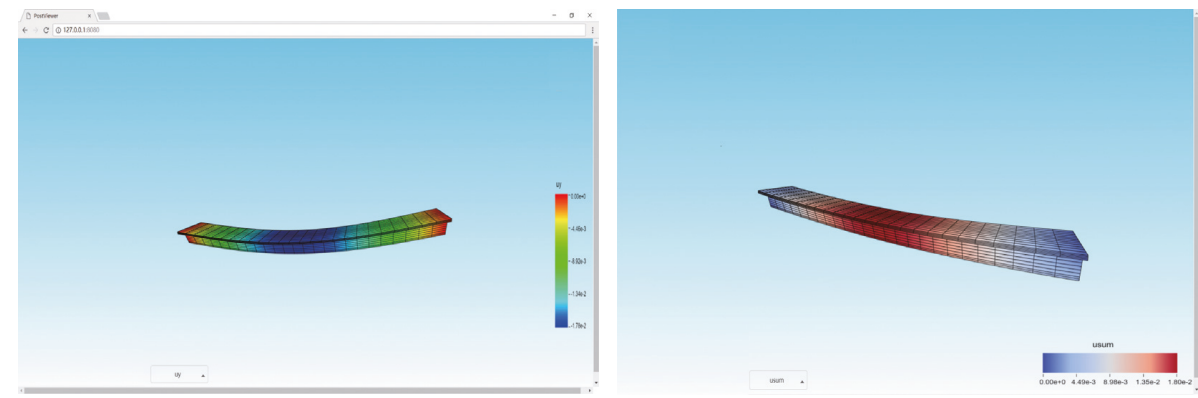

FIGURE 14: Cloud image of node displacement in $\mathrm{U}_{y} /$ sum direction.
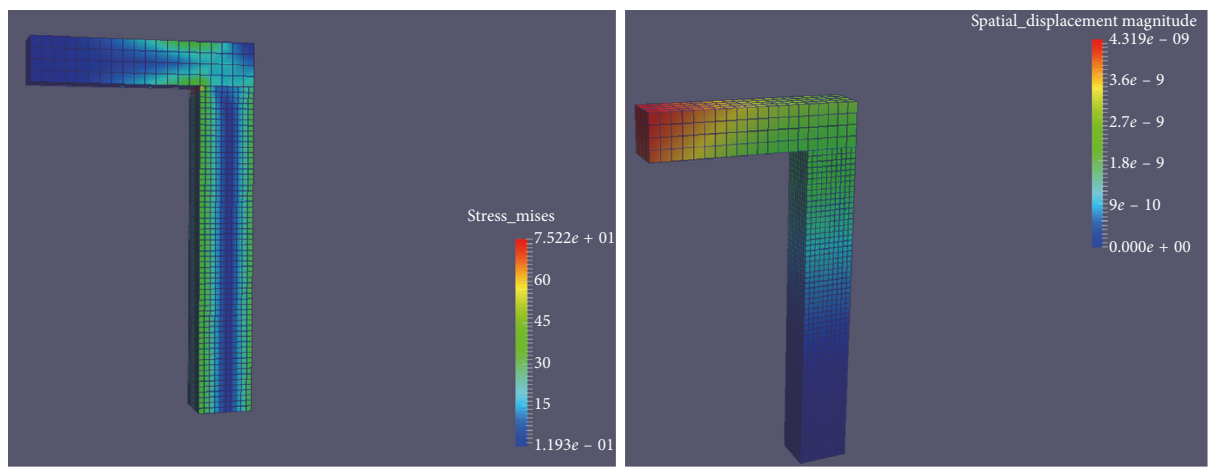

Figure 15: Stress and strain diagrams of frame beams and column grids.

scalar field according to the corresponding relations of these subsurfaces.

(2) Expansion of Entity Type into the Finite Element Model. In the process of transforming the building model to connection model, other solid construction methods can be extended to conduct finite element mesh division. If the building model can be generated and optimized with different algorithms according to different entity expression types, the use efficiency of the building model will be greatly improved, especially for buildings and bridges with complex grids. Moreover, the application of h-p finite element method can also be greatly improved.

(3) Grid Generation of IFC Files in Specific Algorithms. In this paper, the information of IFC files is analyzed and the necessary information in the structural analysis is obtained. In future studies, direct grid compilation of IFC files will become a more critical method. Grid generation can be achieved by directly writing the coordinate information of points in IFC files or by means of entities. This paper mainly manually segments IFC components into grids, which seems not inefficient. At present, not enough research has been devoted on analyzing IFC documents, and the integrated study in the field of structure still needs to explore new methods to expand the application level of BIM technology.

\section{Conclusion}

This paper mainly focuses on IFC standard finite element structure analysis. Considering the advantages of BRep entities, this study uses the BRep model to express all geometric entities through the transformation of the 3D ACIS model. The entity is segmented into the finite element mesh applying the mesh refinement algorithm, and the finite element information of the structure is obtained based on step format data. Through the analysis and expression of IFC files, the finite element analysis can be finally displayed in the IFC standard.

In the transformation from the architectural model to the geometric model, it was found that the entity structure model with BRep entities had unique advantages. The model is transformed into a connected model by analyzing the structure of the model, and the mesh of the model is divided into hexahedrons by the calculation method based on the sweep method. In the process of transformation, although the local construction precision is not enough, the error can be held within the scope of modification. In the field of BIM information, relevant requirements are collected for component extraction firstly to ensure that a single component can be selected for analysis and calculation. The information of the nodes in the IFC file is defined and analyzed to provide necessary data for the finite element analysis. The IFC information was expanded and expressed on the BIM port, and the connection between the IFC and the database was established to ensure that the finite element analysis results were displayed in the IFC standard-based software. In this case, a section of the simply supported beam bridge is 
analyzed and processed, and the displayed results can be visualized in the BIM software. The main contribution of this paper is to develop a method to transfer architecture model to finite element model effectively. The grid model is obtained by meshing IFC files and the results of finite element analysis and then is displayed based on the extension of IFC standards. In this process, the extraction of building components can be completed to provide effective support for structural analysis. In the outlook, it puts forward several directions for future research, which will also provide new thinking for future research in related fields.

\section{Data Availability}

The data used to support the findings of this study are available from the corresponding author upon request.

\section{Conflicts of Interest}

The authors declare that they have no conflicts of interest.

\section{Acknowledgments}

The authors' special thanks go to all survey participants and appreciation goes to the National Science Council of P. R. C. for financially supporting this research (NSFC-71302138).

\section{References}

[1] R. Romberg, A. Niggl, C. van Treeck, and E. Rank, "Structural analysis based on the product model standard IFC," in Proceedings of the ICCCBE, Xth International Conference on Computing in Civil and Building Engineering, Weimar, Germany, July 2004.

[2] C. V. Treeck and E. Rank, "Dimensional reduction of 3D building models using graph theory and its application in building energy simulation," Engineering with Computers, vol. 23, no. 2, pp. 109-122, 2007.

[3] P. Fazio, H. S. He, A. Hammad, and M. Horvat, "IFC-based framework for evaluating total performance of building envelopes," Journal of Architectural Engineering, vol. 13, no. 1, pp. 44-53, 2007.

[4] H. Ying and S. Lee, "A framework for rule-based validation of IFC space boundaries for building energy analysis," in Proceedings of the ASCE International Workshop on Computing in Civil Engineering 2017, Seattle, Washington, June 2017.

[5] M. H. Serror, J. Inoue, Y. Adachi, and Y. Fujino, "Shared computer-aided structural design model for construction industry (infrastructure)," Computer-Aided Design, vol. 40, no. 7, pp. 778-788, 2008.

[6] B. Hatipoglu and C. Ozturan, "Parallel triangular mesh refinement by longest edge bisection," SIAM Journal on Scientific Computing, vol. 37, no. 5, pp. C574-C588, 2015.

[7] K. Kähler, J. Haber, and H. P. Seidel, "Geometry-based muscle modeling for facial animation," in Proceedings of Graphics Interface 2001, Ottawa, Ontario, Canada, June 2001.

[8] Y. K. Lee and C. K. Lee, "A new indirect anisotropic quadrilateral mesh generation scheme with enhanced local mesh smoothing procedures," International Journal for Numerical Methods in Engineering, vol. 58, no. 2, pp. 277-300, 2003.

[9] S. Nagakura, S. Noguchi, H. Yamashita, and V. Cingoski, "Automatic hexahedral mesh generation for FEM using shape recognition technique and tree method [EM field analysis],"
IEEE Transactions on Magnetics, vol. 38, no. 2, pp. 417-420, 2002.

[10] M. W. Lai, S. Benzley, and D. White, "Automated Hexahedral mesh generation by generalized multiple source to multiple target sweeping," International Journal for $\mathrm{Nu}$ merical Methods in Engineering, vol. 49, no. 1-2, pp. 261-275, 2015.

[11] A. C. D. Miranda and L. F. Martha, "Hierarchical templatebased hexahedral mesh generation," Engineering with Computers, vol. 34, no. 3, pp. 465-474, 2018.

[12] M. Elhaddad, N. Zander, T. Bog et al., "Multi-level hp-finite cell method for embedded interface problems with application in biomechanics," International Journal for Numerical Methods in Biomedical Engineering, vol. 34, no. 4, pp. 29-51, 2018.

[13] T. Liebich, IFC4-the New Building SMART Standard, IC Meeting, BSI Publications, Helsinki, Finland, 2013.

[14] R. Hitchcock and J. Wong, "Transforming IFC architectural view BIMs for energy simulation," in Proceedings of the Building Simulation IBPSA Conference, pp. 1089-1095, Sydney, Australia, November 2011.

[15] T. Krijnen and J. Beetz, "A SPARQL query engine for binaryformatted IFC building models," Automation in Construction, vol. 95, pp. 46-63, 2018.

[16] Z. Xu, T. Huang, B. Li, H. Li, and Q. Li, "Developing an IFCbased database for construction quality evaluation," Advances in Civil Engineering, vol. 2018, pp. 1-22, 2018.

[17] G. N. Lilis, G. I. Giannakis, and D. V. Rovas, "Automatic generation of second-level space boundary topology from IFC geometry inputs," Automation in Construction, vol. 76, pp. 108-124, 2017.

[18] S. Daum and A. Borrmann, "Processing of topological BIM queries using boundary representation based methods," Advanced Engineering Informatics, vol. 28, no. 4, pp. 272-286, 2014.

[19] T. W. Kang and C. H. Hong, "IFC-CityGML LOD mapping automation using multiprocessing-based screen-buffer scanning including mapping rule," KSCE Journal of Civil Engineering, vol. 22, no. 2, pp. 16-19, 2017.

[20] N. Yabuki, "Representation of caves in a shield tunnel product model," in Proceedings of the 7th European Conference on Product and Process Modeling, Sophia Antipolis, France, September 2018.

[21] S. H. Lee and B. G. Kim, "IFC Extension for Road Structures and Digital Modeling," in Proceedings of the Twelfth East AsiaPacific Conference on Structural Engineering \& Construction, Hong Kong, China, January 2011.

[22] S.-H. Lee, S. I. Park, and J. Park, "Development of an IFCbased data schema for the design information representation of the NATM tunnel," KSCE Journal of Civil Engineering, vol. 20, no. 6, pp. 2112-2123, 2016.

[23] S. Park, J. Park, B.-G. Kim, and S.-H. Lee, "Improving applicability for information model of an IFC-based steel bridge in the design phase using functional meanings of bridge components," Applied Sciences, vol. 8, no. 12, pp. 2531-2562, 2018.

[24] L. Barazzetti, F. Banfi, R. Brumana, G. Gusmeroli, M. Previtali, and G. Schiantarelli, "Cloud-to-BIM-to-FEM: structural simulation with accurate historic BIM from laser scans," Simulation Modelling Practice and Theory, vol. 57, pp. 71-87, 2015.

[25] R. C. Xiao, Y. Lian, B. Sun, X. W. Zhao, Z. Liu, and P. B. Tang, "Method of bridge structural analysis based on bridge information modeling," in Proceedings of the Computing in Civil 
Engineering 2017: Smart Safety, Sustainability and Resilience-Selected Papers from the ASCE International Workshop on Computing in Civil Engineering, pp. 92-100, Seattle, Washington, June 2017.

[26] M. Huang, Y. Du, J. Zhang, and Y. Zhang, "A topological enabled three-dimensional model based on constructive solid geometry and boundary representation," Cluster Computing, vol. 19, no. 4, pp. 2027-2037, 2016.

[27] B. Stefano, M. S. Emilio, S. Aleksandra, and T. Walter, "Towards an ontological grounding of IFC," in Proceedings of the 6th Workshop on Formal Ontologies Meet Industry (FOMI), Rio de Janeiro, Brasil, September 2014.

[28] K. P. Karunakaran and R. Shringi, "Octree-to-BRep conversion for volumetric NC simulation," The International Journal of Advanced Manufacturing Technology, vol. 32, no. 12, pp. 116-131, 2007.

[29] A. Düster, S. Hartmann, and E. Rank, "p-FEM applied to finite isotropic hyperelastic bodies," Computer Methods in Applied Mechanics and Engineering, vol. 192, no. 47-48, pp. 51475166, 2003. 


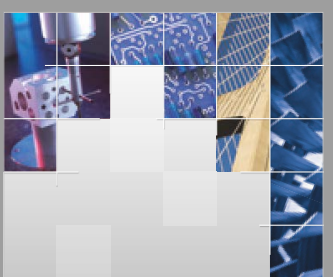

\section{Enfincering}
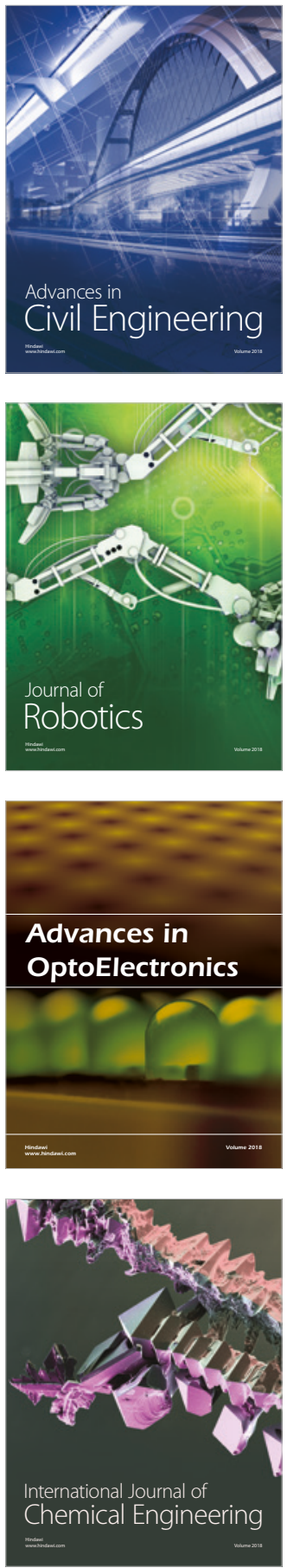

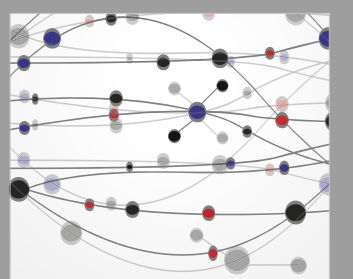

\section{Rotating \\ Machinery}

The Scientific World Journal

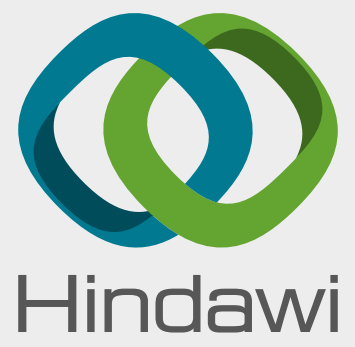

Submit your manuscripts at

www.hindawi.com
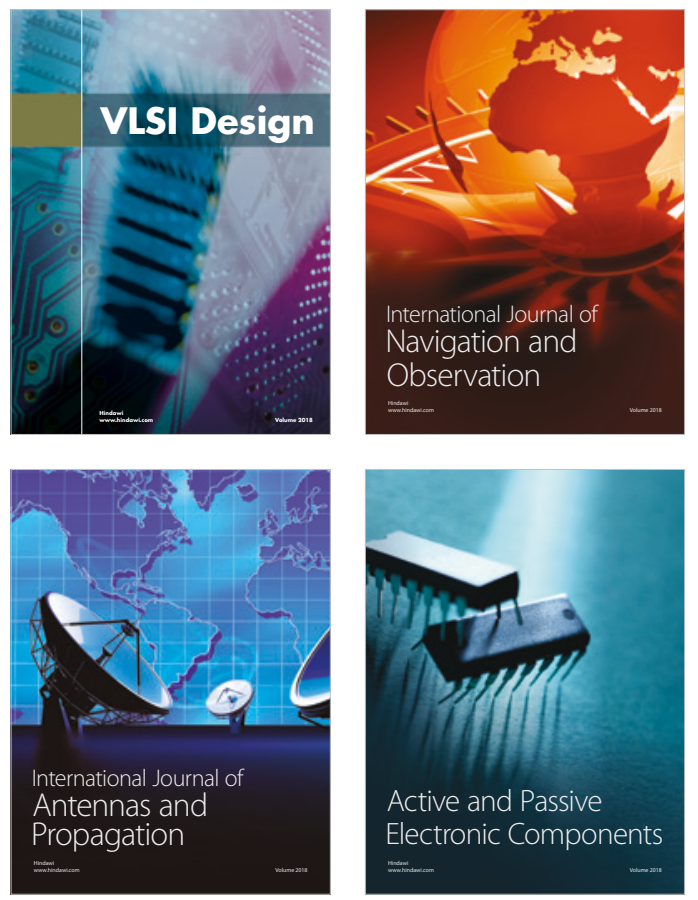
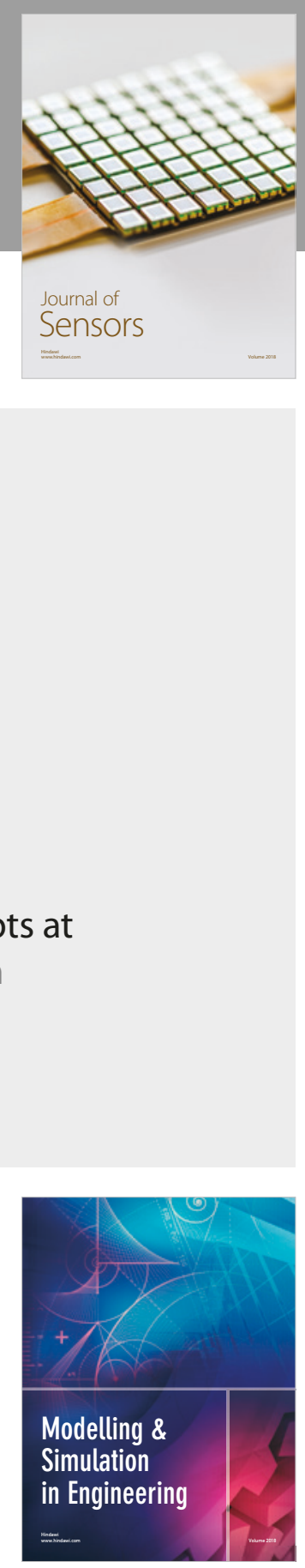

\section{Advances \\ Multimedia}
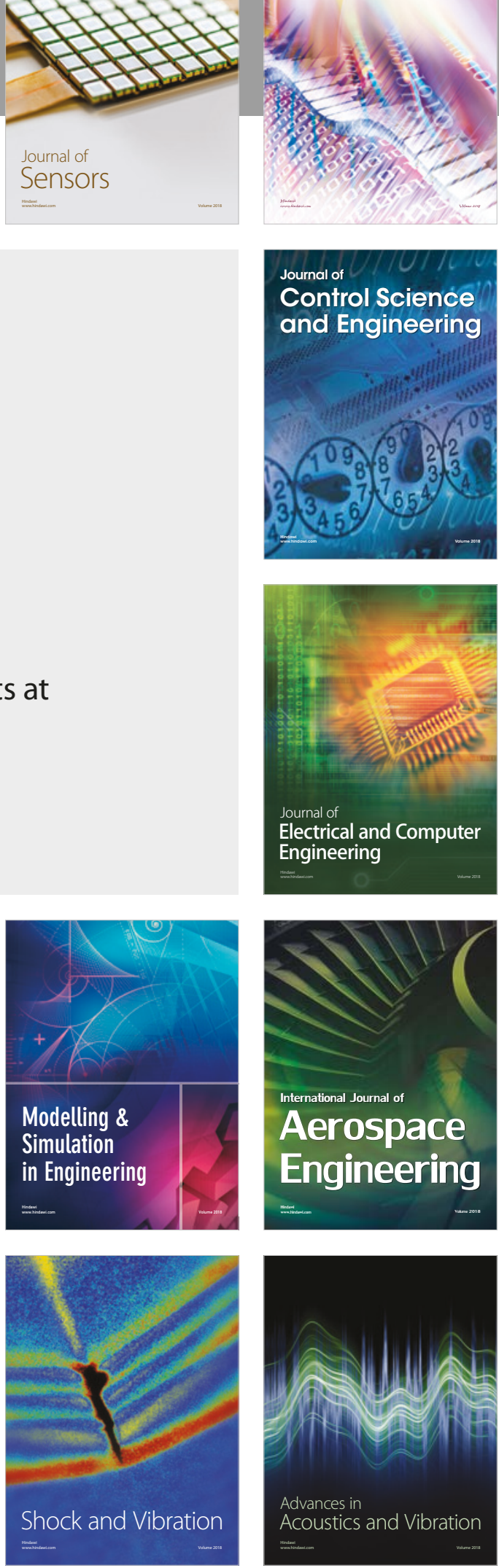